\title{
CARDINAL HERMITE SPLINE INTERPOLATION: CONVERGENCE AS THE DEGREE TENDS TO INFINITY(')
}

\author{
BY
}

\author{
M. J. MARSDEN AND S. D. RIEMENSCHNEIDER
}

\begin{abstract}
Let $\delta_{2 m, r}$ denote the class of cardinal Hermite splines of degree $2 m-1$ having knots of multiplicity $r$ at the integers. For $f(x) \in C^{r-1}(R)$, the cardinal Hermite spline interpolant to $f(x)$ is the unique element of $S_{2 m, r}$ which interpolates $f(x)$ and its first $r-1$ derivatives at the integers. For $y=\left(y^{0}, \ldots, y^{r-1}\right)$ an $r$-tuple of doubly-infinite sequences, the cardinal Hermite spline interpolant to $y$ is the unique $S(x) \in \mathcal{S}_{2 m, r}$ satisfying $S^{(s)}(v)$ $=y^{s}, s=0,1, \ldots, r-1$, and $v$ an integer.

The following results are proved: If $f(x)$ is a function of exponential type less than $m$, then the derivatives of the cardinal Hermite spline interpolants to $f(x)$ converge uniformly to the respective derivatives of $f(x)$ as $m \rightarrow \infty$. For functions from more general, but related, classes, weaker results hold. If $y$ is an $r$-tuple of $l^{p}$ sequences, then the cardinal Hermite spline interpolants to $y$ converge to $W_{r}(y)$, a certain generalization of the Whittaker cardinal series which lies in the Sobolev space $W^{p, r-1}(R)$. This convergence is in the Sobolev norm.

The class of all such $W_{r}(y)$ is characterized. For small values of $r$, the explicit forms of $W_{r}(y)$ are described.
\end{abstract}

Let $r$ and $m$ be natural numbers such that $r \leqslant m$, and let $\delta_{2 m, r}$ denote the class of functions, $S(x)$, satisfying: $S(x)$ is a polynomial of degree $2 m-1$ on each interval $[\nu, \nu+1], \nu \in \mathbf{Z}$; and $S(x) \in C^{2 m-r-1}(\mathbf{R})$. That is, $\delta_{2 m, r}$ is the class of spline functions of degree $2 m-1$ with knots at the integers of multiplicity $r$.

Given an $r$-tuple of sequences, $\left(y^{0}, \ldots, y^{r-1}\right), y^{s}=\left\{y_{v}^{s}: \nu \in \mathbf{Z}\right\}$, the cardinal Hermite interpolation problem is to find an (unique) element $S(x)$ $\in \mathcal{S}_{2 m, r}$ such that $S^{(s)}(\nu)=y_{v}^{s}, s=0,1, \ldots, r-1$, and $\nu \in \mathbf{Z}$. P. Lipow and I. J. Schoenberg [4] established that if the sequences are of power growth, i.e. $y_{\nu}=\theta\left(|\nu|^{\gamma}\right)$ as $|\nu| \rightarrow \infty$, then there is a unique spline $S(x) \in \delta_{2 m, r}$ that solves the interpolation problem and is of power growth, $|S(x)|=\theta\left(|x|^{\gamma}\right)$ as $|x| \rightarrow$ $\infty$. Moreover, this spline is given by the formula

Received by the editors June 28, 1976.

AMS (MOS) subject classifications (1970). Primary 41A15; Secondary 42A68.

Key words and phrases. Cardinal Hermite splines, Hermite interpolation, Whittaker series, summability, Sobolev spaces.

(') This research is partially supported by Canadian National Research Council Grant A-7687. 


$$
e_{2 m, r}\left(y^{0}, \ldots, y^{r-1}\right)(x)=\sum_{s=0}^{r-1} \sum_{\nu \in Z} y_{\nu}^{s} L_{2 m, r, s}(x-\nu)
$$

where the fundamental splines $L_{2 m, r, s}(x)$ are the unique bounded splines determined by $L_{2 m, r, s}^{(\rho)}(0)=\delta_{\rho, s}$, and $L_{2 m, r, s}^{(\rho)}(\nu)=0, \nu \neq 0, \rho=0,1, \ldots, r-$ 1.

If $l^{p} \oplus \cdots \oplus_{r} l^{p}$ denotes the Banach space of $r$-tuples, $\left(y^{0}, \ldots, y^{r-1}\right)$, of $l^{p}$ sequences with norm $\Sigma_{s}\left\|y^{s}\right\|_{l p}$, and $W^{p, r-1}(\mathbf{R})$ denotes the Sobolev space of functions $f$ for which $f^{(r-2)}$ is absolutely continuous and $f^{(s)} \in L^{p}(\mathbf{R})$, $s=0, \ldots, r-1$, with the norm $\|f\|_{p, r-1}=\Sigma_{s}\left\|f^{(s)}\right\|_{L^{p}}$, then Lipow and Schoenberg [4] have shown that $\mathfrak{L}_{2 m, r}$ is a linear operator from $l^{p}$ $\oplus \cdots \oplus_{r} l^{p}$ to $W^{p, r-1}(\mathbf{R}), 1 \leqslant p \leqslant+\infty$.

The purpose of the present paper is to study the following convergence problems: (1) For what suitably restricted class of functions do the cardinal Hermite splines interpolating any one of the functions and its derivatives to order $r-1$ at the integers converge to the function as the degree tends to infinity, and in what sense is the convergence? (2) What is the behaviour of the operators $f_{2 m, r}$ as a sequence of mappings from $l^{p} \oplus \cdots \oplus_{r} l^{p}$ to $W^{p, r-1}$, and what is the description of the limiting operator if one exists?

In the case of single knots $(r=1)$, these and similar problems have been studied by a number of authors ([2], [5], [6], [9]-[19]). In the monograph [15, p. 107], Schoenberg raises the question as to the existence of a comprehensive theory that would cover the various known cases of convergence $(r=1)$. The Fourier transform approach employed in [6] and [13] provides a method which yields most of the analogous convergence results for the multiple knot case.

The method requires the study of the Fourier transforms of fundamental splines. Recently, S. L. Lee [3] has established the representation

$$
L_{2 m, r, s}(x)=\frac{(-i)^{s}}{2 \pi} \int_{-\infty}^{+\infty} H_{2 m, r, s}(u) e^{i u x} d u,
$$

where $H_{2 m, r, s}(u)=H_{r, s}\left(\alpha_{2 m}(u)\right) / H_{r}\left(\alpha_{2 m}(u)\right)$, with $H_{r}\left(\alpha_{n}\right)$ being the Hankel determinant defined by

$$
H_{r}\left(\alpha_{n}\right)=\left|\begin{array}{llll}
\alpha_{n} & \alpha_{n-1} & \cdots & \alpha_{n-r+1} \\
\alpha_{n-1} & \alpha_{n-2} & \cdots & \alpha_{n-r} \\
\vdots & \vdots & & \vdots \\
\alpha_{n-r+1} & \alpha_{n-r} & & \alpha_{n-2 r+2}
\end{array}\right|,
$$

$\alpha_{n}(u)=\Sigma_{k \in \mathbf{Z}}(u+2 \pi k)^{-n}$, and $H_{r, s}\left(\alpha_{2 m}(u)\right)$ is formed from $H_{r}\left(\alpha_{2 m}(u)\right)$ by replacing the $(s+1)$ st column by the column vector $\left(u^{-2 m}\right.$, $\left.u^{-2 m+1}, \ldots, u^{-2 m+r-1}\right)^{T}$. 
In $\S 1$, we find another representation for $H_{2 m, r, s}(u)$ and discuss properties that may be deduced from this representation. In $\$ 2$, we discuss the limit of $H_{2 m, r, s}(u)$ as $m \rightarrow \infty$, the nature of the convergence, and some identities satisfied by the limit function. Because of the nature of $H_{2 m, r, s}(u)$, the proofs in both sections are technically involved but elementary. For this reason, the proofs are fairly detailed until our methods are established. The reader should reflect on the representation and the assertions since the course of action is far more obvious than the calculations.

The hard work necessary for the convergence theorems is done in the first two sections and the remainder of the paper is devoted to applications of these results in methods largely developed elsewhere. In \$3, we obtain the answer to question (1) above for functions which are Fourier-Stieltjes transforms of measures on $[-r \pi, r \pi)$ and uniform convergence. Some additional results are obtained when the functions are Fourier transforms of certain distributions with support in $(-r \pi, r \pi)$ and the convergence is uniform on bounded subsets of $\mathbf{R}$.

The fourth section completely answers the second question above when $1<p<+\infty$. As a consequence, the first question is answered by specifying the class of functions in the Sobolev space $W^{p, r-1}(\mathbf{R})$ for which the convergence is in the $W^{p, r-1}(\mathbf{R})$ norm, $1<p<\infty$. The final section is devoted to some examples.

Some of these results were presented at the Symposium on Approximation Theory at Austin in January 1976 (see [8]).

1. Properties of $H_{2 m, r, s}(u)$. The first results are technical lemmas that make the dominant terms of $H_{2 m, r_{,},}(u)$ more visible.

Let $V\left(\alpha_{1}, \ldots, \alpha_{n}\right)=\Pi_{1<j<k<n}\left(\alpha_{k}-\alpha_{j}\right)$ denote the Vandermonde of $\alpha_{1}, \ldots, \alpha_{n}$.

LEMMA 1.1. If $u / 2 \pi$ is not an integer, then

$$
\begin{aligned}
& H_{r}\left(\alpha_{2 m}(u)\right) \\
& \quad=\sum_{i_{1}<i_{2}<\cdots<i_{r}} \frac{(2 \pi)^{r(r-1)} V^{2}\left(i_{1}, \ldots, i_{r}\right)}{\Pi_{1<j<r}\left(u+2 \pi i_{j}\right)^{2 m}} .
\end{aligned}
$$

Proof. Let $F\left(i_{1}, \ldots, i_{n}\right)=\Pi_{1<j<n}\left(u+2 \pi i_{j}\right)^{j-1}$. Let $P\left(i_{1}, \ldots, i_{n}\right)$ be an arbitrary permutation of $i_{1}, \ldots, i_{n}$. Let $\sigma(P)=+1(-1)$ if $P$ is even (odd). Let $\Sigma_{P\left(i_{1}, \ldots, i_{n}\right)} f(P)$ be the summation of $f\left(P\left(i_{1}, \ldots, i_{n}\right)\right)$ over all permutations of $i_{1}, \ldots, i_{n}$.

From the representation (0.2), 


$$
\begin{aligned}
& H_{r}\left(\alpha_{2 m}(u)\right)=\sum_{i_{1}, \ldots, i_{r}} \frac{\left|\begin{array}{ccc}
1 & \cdots & 1 \\
u+2 \pi i_{1} & \cdots & u+2 \pi i_{r} \\
\vdots & & \vdots \\
\left(u+2 \pi i_{1}\right)^{r-1} & \cdots & \left(u+2 \pi i_{r}\right)^{r-1}
\end{array}\right|}{\left(u+2 \pi i_{1}\right)^{2 m}\left(u+2 \pi i_{2}\right)^{2 m-1} \cdots\left(u+2 \pi i_{r}\right)^{2 m-r+1}} \\
& =\sum_{i_{1}, \ldots, i_{r}} \frac{V\left(u+2 \pi i_{1}, \ldots, u+2 \pi i_{r}\right) F\left(i_{1}, \ldots, i_{r}\right)}{\left(u+2 \pi i_{1}\right)^{2 m}\left(u+2 \pi i_{2}\right)^{2 m} \cdots\left(u+2 \pi i_{r}\right)^{2 m}} \\
& =\sum_{i_{1}, \ldots, i_{r}} \frac{(2 \pi)^{r(r-1) / 2} V\left(i_{1}, \ldots, i_{r}\right) F\left(i_{1}, \ldots, i_{r}\right)}{\left(u+2 \pi i_{1}\right)^{2 m}\left(u+2 \pi i_{2}\right)^{2 m} \cdots\left(u+2 \pi i_{r}\right)^{2 m}} \\
& =\sum_{i_{1}<\cdots<i_{r}} \frac{(2 \pi)^{r(r-1) / 2}}{\Pi_{1<j<r}\left(u+2 \pi i_{j}\right)^{2 m}}\left[\sum_{P\left(i_{1}, \ldots, i_{r}\right)} V(P) F(P)\right] \\
& =\sum_{i_{1}<\cdots<i_{r}} \frac{(2 \pi)^{r(r-1) / 2} V\left(i_{1}, \ldots, i_{r}\right)}{\Pi_{1<j<r}\left(u+2 \pi i_{j}\right)^{2 m}}\left[\sum_{P\left(i_{1}, \ldots, i_{r}\right)} \sigma(P) F(P)\right] \\
& =\sum_{i_{1}<\cdots<i_{r}} \frac{(2 \pi)^{r(r-1) / 2} V\left(i_{1}, \ldots, i_{r}\right)}{\Pi_{1<j \leqslant r}\left(u+2 \pi i_{j}\right)^{2 m}} V\left(u+2 \pi i_{1}, \ldots, u+2 \pi i_{r}\right) \\
& =\sum_{i_{1}<\cdots<i_{r}} \frac{(2 \pi)^{r(r-1)} V^{2}\left(i_{1}, \ldots, i_{r}\right)}{\prod_{1<j<r}\left(u+2 \pi i_{j}\right)^{2 m}} \text {. }
\end{aligned}
$$

Before deriving similar expressions for $H_{r, s}\left(\alpha_{2 m}(u)\right)$ and $H_{2 m, r, s}(u)$, it is convenient to introduce some notation:

(1.1) $\quad l=l(u)$ and $x=x(u)$ will be given by the constraints: $2 l+r$ is an odd integer and $-\frac{1}{2}<x=(u-2 \pi l) / 2 \pi<\frac{1}{2}$.

(i) will denote an arbitrary set $i_{1}, \ldots, i_{\text {r }}$ of integers or (1.2) half-odd-integers satisfying: $2 i_{j}+r$ is an odd integer for $j=1, \ldots, r ; i_{1}<i_{2}<\cdots<i_{s} ; i_{s+1}=l ; i_{s+2}<\cdots<$ $i_{r}$; and all $i_{j}$ are distinct.

$k$ will denote an arbitrary set $k_{1}, \ldots, k_{r}$ of integers or

(1.3) half-odd integers satisfying: $2 k_{j}+r$ is an odd integer for $j=1, \ldots, r$ and $k_{1}<\cdots k_{r}$.

(1.4) $\quad \eta$ will denote the set $\eta_{1}, \eta_{2}, \ldots, \eta_{r}$ satisfying $\eta_{j}=j-(r+$ 1) $/ 2$ for $j=1, \ldots, r$. 


$$
\begin{aligned}
& V(i)=V\left(i_{1}, \ldots, i_{r}\right), V_{1}(i)=V\left(i_{1}, \ldots, i_{s}\right), \quad V_{2}(i)= \\
& V\left(i_{s+2}, \ldots, i_{r}\right), \quad V(k)=V\left(k_{1}, \ldots, k_{r}\right), V(\eta)= \\
& V\left(\eta_{1}, \ldots, \eta_{r}\right) .
\end{aligned}
$$

By $\Sigma_{(i)}$ we shall mean summation over all $r$-tuples $(i)=\left(i_{1}, \ldots, i_{r}\right)$ of the type described in (1.2). By $\Sigma_{k}$ we shall mean summation over all $r$-tuples $k=\left(k_{1}, \ldots, k_{r}\right)$ of the type described in (1.3).

Our choice of $(i), k, \eta$, and $l$ as sets of integers (half-odd-integers) when $r$ is odd (even) is ultimately dictated by the fact that

$$
Q_{r, s}(u)=\lim _{m \rightarrow \infty} H_{2 m, r, s}(u)
$$

is itself a Hermite spline with knot intervals of length $2 \pi$ centered at integer (half-odd-integer) multiples of $2 \pi$ when $r$ is odd (even). For the present, however, this choice is a convenience which simplifies the proofs of Lemmas 1.4 and 1.5 below.

Finally, the statement $(i)=k$ will mean that (i) satisfies (1.2), $k$ satisfies (1.3), and that $(i)$ and $k$ are equal when considered as unordered sets. The statement $(i) \neq k$ will mean that at least one element is different. A similar definition applies when $\eta$ is compared with (i) or $k$.

LEMMA 1.2. If $u / 2$ is not an integer, then

$$
\begin{aligned}
& H_{r, s}\left(\alpha_{2 m}(u)\right) \\
& \quad=\sum_{(i)} \frac{(2 \pi)^{t} V(i) V_{1}(i) V_{2}(i)}{\Pi_{1<j<s+1}\left(u-2 \pi l+2 \pi i_{j}\right)^{2 m} \Pi_{s+2<j<r}\left(u-2 \pi l+2 \pi i_{j}\right)^{2 m-s-1}}
\end{aligned}
$$

where

$$
t=\left[r^{2}-r+s^{2}-s+(r-s-1)(r-s-2)\right] / 2 .
$$

Proof. For a given ( $i$ ), let $P_{1}$ denote an arbitrary permutation of $i_{1}, \ldots, i_{s}$, let $P_{2}$ denote an arbitrary permutation of $i_{s+2}, \ldots, i_{r}$, let $\sigma\left(P_{1}\right)$ and $\sigma\left(P_{2}\right)$ be their respective parities, and let $\Sigma_{1,2}$ denote summation over all such pairs $P_{1}$ and $P_{2}$. Let $\Sigma_{(i, 0)}$ denote summation over all $r$-tuples like those described in (1.2) except that $i_{s+1}=0$ instead of $i_{s+1}=l$ and each $i_{j}$ is an integer.

From the representation (0.2) and minor changes in the proof of Lemma 1.1 , 


$$
\begin{aligned}
H_{r, s}\left(\alpha_{2 m}(u)\right) & =\sum_{\substack{i_{1}, \ldots, i_{r} \\
i_{s+1}=0}} \frac{(2 \pi)^{r(r-1) / 2} V\left(i_{1}, \ldots, i_{r}\right)}{u^{s}\left(u+2 \pi i_{1}\right)^{2 m}\left(u+2 \pi i_{2}\right)^{2 m-1} \cdots\left(u+2 \pi i_{r}\right)^{2 m-r+1}} \\
& =\sum_{\substack{i_{1}, \ldots, i_{r}, i_{s+1}=0}} \frac{(2 \pi)^{r(r-1) / 2} V(i) F\left(i_{1}, \ldots, i_{s}\right) F\left(i_{s+2}, \ldots, i_{r}\right)}{\Pi_{1<j<s+1}\left(u+2 \pi i_{j}\right)^{2 m} \Pi_{s+2<j<r}\left(u+2 \pi i_{j}\right)^{2 m-s-1}} \\
& =\sum_{(i, 0)} \frac{(2 \pi)^{r(r-1) / 2} \sum_{1,2} V\left(P_{1}, 0, P_{2}\right) F\left(P_{1}\right) F\left(P_{2}\right)}{\Pi_{1<j<s+1}\left(u+2 \pi i_{j}\right)^{2 m} \Pi_{s+2<j<r}\left(u+2 \pi i_{j}\right)^{2 m-s-1}} \\
& =\sum_{(i, 0)} \frac{(2 \pi)^{r(r-1) / 2} V(i) \sum_{1,2} \sigma\left(P_{1}\right) F\left(P_{1}\right) \sigma\left(P_{2}\right) F\left(P_{2}\right)}{\Pi_{1<j<s+1}\left(u+2 \pi i_{j}\right)^{2 m} \Pi_{s+2<j<r}\left(u+2 \pi i_{j}\right)^{2 m-s-1}} \\
& =\sum_{(i, 0)} \frac{(2 \pi)^{r(r-1) / 2} V(i) V\left(u+2 \pi i_{1}, \ldots, u+2 \pi i_{s}\right) V\left(u+2 \pi i_{s+2}, \ldots, u+2 \pi i_{r}\right)}{\Pi_{1<j<s+1}\left(u+2 \pi i_{j}\right)^{2 m} \Pi_{s+2<j<r}\left(u+2 \pi i_{j}\right)^{2 m-s-1}} \\
& =\sum_{(i, 0)} \frac{(2 \pi)^{t} V(i) V_{1}(i) V_{2}(i)}{\Pi_{1<j<s+1}\left(u+2 \pi i_{j}\right)^{2 m} \Pi_{s+2<j<r}\left(u+2 \pi i_{j}\right)^{2 m-s-1}} \cdot
\end{aligned}
$$

The result follows from the replacement $i_{j} \rightarrow i_{j}-l$ where $l$ is the integer or half-odd-integer described in (1.1).

Lemmas 1.1 and 1.2 give the following representation:

Proposmion 1.3. Let $k$ be described by (1.3). For any $u \in \mathbf{R}$, let $l, x$, and (i) be described by (1.1) and (1.2). Then

$$
\begin{aligned}
& H_{2 m, r, s}(u) \\
& =\frac{\sum_{(i)}\left(V(i) V_{1}(i) V_{2}(i) / \Pi_{1<j<s+1}\left(x+i_{j}\right)^{2 m} \Pi_{s+2<j<r}\left(x+i_{j}\right)^{2 m-s-1}\right)}{(2 \pi)^{s} \Sigma_{k}\left(V^{2}(k) / \Pi_{1<j<r}\left(x+k_{j}\right)^{2 m}\right)} .
\end{aligned}
$$

In (1.6), we assume that the singularities at $u=0, \pm 2 \pi, \pm 4 \pi, \ldots$ have been removed. Since $m>r$, the series converge uniformly and $H_{2 m, r, s}(u)$ is a continuous function.

LEMMA 1.4. As $m \rightarrow \infty, H_{2 m, r, s}(u)=\theta\left(\min \left(1,(r \pi / u)^{2 m}\right)\right)$

Proof. By deleting (positive) terms in the dominator of (1.6), we have 
$H_{2 m, r, s}(u)$

$$
\begin{aligned}
& <\frac{\sum_{(i)}\left(|V(i)| V_{1}(i) V_{2}(i) / \Pi_{1<j<s+1}\left(x+i_{j}\right)^{2 m} \Pi_{s+2<j<r}\left|x+i_{j}\right|^{2 m-s-1}\right)}{(2 \pi)^{s} V^{2}(\eta) \Pi_{1<j<r}\left(x+\eta_{j}\right)^{-2 m}} \\
& =\sum_{(i)} W_{r, s}(x, i) \prod_{1<j<r}\left[\frac{\left(x+\eta_{j}\right)}{\left(x+i_{j}\right)}\right]^{2 m}
\end{aligned}
$$

where $\eta$ is given by (1.4) and

$$
\begin{aligned}
W_{r, s}(x, i) & =|V(i)| V_{1}(i) V_{2}(i) \prod_{s+2<j<r} \frac{\left|x+i_{j}\right|^{s+1}}{(2 \pi)^{s} V^{2}(\eta)} \\
& <|V(i)| V_{1}(i) V_{2}(i) \prod_{s+2<j<r}\left(\left|i_{j}\right|+1\right)^{s+1} .
\end{aligned}
$$

The right side of (1.8) is a polynomial expression of degree $<2 r-2$ in each $i_{j}$ whose coefficients are independent of $m$.

For each $(i)$ entering the above sum, let $P(i)=\left\{P_{1}(i), P_{2}(i), \ldots, P_{r}(i)\right\}$ be the permutation of $(i)$ satisfying

$$
\left|x+P_{1}(i)\right|<\left|x+P_{2}(i)\right|<\cdots<\left|x+P_{r}(i)\right| \text {. }
$$

Similarly, let $Q(\eta)=\left(Q_{1}(\eta), \ldots, Q_{r}(\eta)\right)$ order the $\left|x+\eta_{j}\right|$. Setting $S=$ $P^{-1} Q$, we have

$$
\left|H_{2 m, r, s}(u)\right|<\mid \sum_{(i)} W_{r, s}(x, i) \prod_{1<j<r}\left[\frac{\left(x+S_{j}(\eta)\right)}{\left(x+i_{j}\right)}\right]^{2 m}
$$

In view of our choice of $S$ and the fact that $\eta$ is "closest" to $-x$,

$$
\left|\left(x+S_{j}(\eta)\right) /\left(x+i_{j}\right)\right|<1 \text { for all } j .
$$

In addition, if $\left|i_{j}\right|>(r-1) / 2$,

$$
\left|\left(x+S_{j}(\eta)\right) /\left(x+i_{j}\right)\right|<(r / 2) /\left|i_{j}+x\right|<\left(r /\left(2\left|i_{j}\right|-1\right)\right) .
$$

Thus,

(1.9) $\left|\left(x+S_{j}(\eta)\right) /\left(x+i_{j}\right)\right|<\min \left(1,\left|r /\left(2\left|i_{j}\right|-1\right)\right|\right)=a_{r}\left(i_{j}\right)$.

The above discussion will be referred to as the "pairing argument" in the sequel.

Combining equations (1.7), (1.8) and (1.9) gives

$$
\begin{aligned}
\left|H_{2 m, r, s}(u)\right| & <\sum_{(i)} W_{r, s}(x, i) \prod_{1<j<r}\left[a_{r}\left(i_{j}\right)\right]^{2 m} \\
& <\sum_{(i)} W_{r, s}(x, i) \prod_{1<j<r}\left[a_{r}\left(i_{j}\right)\right]^{2 r}<C_{r, 1}
\end{aligned}
$$


since $m>r$. Thus, $H_{2 m, r, s}(u)=\mathcal{O}(1)$ as $m \rightarrow \infty$.

Now, suppose that $|u|>r \pi$. Then

$$
\left|\frac{x+S_{s+1}(\eta)}{x+i_{s+1}}\right|=\left|\frac{x+S_{s+1}(\eta)}{x+l}\right| \leqslant\left|\frac{r / 2}{u / 2 \pi}\right|=\left|\frac{r \pi}{u}\right|
$$

so that

$$
\begin{aligned}
\left|H_{2 m, r, s}(u)\right| & \leqslant\left(\frac{r \pi}{u}\right)^{2 m} \sum_{(i)} W_{r, s}(x, i) \prod_{j \neq s+1}\left[a_{r}\left(i_{j}\right)\right]^{2 m} \\
& \leqslant C_{r, 2}(r \pi / u)^{2 m}
\end{aligned}
$$

for $m>r$, as before.

LEMMA 1.5. For $\rho=0,1, \ldots, r-1$,

$$
\operatorname{Var}\left(u^{\rho} H_{2 m, r, s}(u)\right)=\int_{-\infty}^{\infty}\left|\frac{d}{d u}\left(u^{\rho} H_{2 m, r, s}(u)\right)\right| d u=\mathcal{O}(1)
$$

as $m \rightarrow \infty$.

Proof. From the product rule and Lemma 1.4,

$$
\operatorname{Var}\left(u^{\rho} H_{2 m, r, s}(u)\right)<\mathcal{O}(1)+\int\left|u^{\rho} H_{2 m, r, s}^{\prime}(u)\right| d u .
$$

For $u / \pi \notin \mathbf{Z}$, we differentiate (1.6) to obtain

$$
=\frac{\sum_{(i)} \Sigma_{k} \frac{V(i) V_{1}(i) V_{2}(i) V^{2}(k) C_{m, r, s}(x, i, k)}{\Pi_{1<j<s+1}\left(x+i_{j}\right)^{2 m} \Pi_{s+2<j<r}\left(x+i_{j}\right)^{2 m-s-1} \Pi_{1<j<r}\left(x+k_{j}\right)^{2 m}}}{(2 \pi)^{s+1}\left[\sum_{k} \frac{V^{2}(k)}{\Pi_{1<j<r}\left(x+k_{j}\right)^{2 m}}\right]^{2}}
$$

where

$$
\begin{aligned}
C_{m, r, s}(x, i, k)= & \sum_{1<j<s+1} \frac{-2 m}{x+i_{j}} \\
& +\sum_{s+2<j<r} \frac{s+1-2 m}{x+i_{j}}+\sum_{1<j<r} \frac{2 m}{x+k_{j}}
\end{aligned}
$$

arises from the terms of $H_{r} H_{r, s}^{\prime}-H_{r}^{\prime} H_{r, s}$.

When $(i)=k$ as sets, the $m$-dependence in $C_{m, r, s}(x, i, k)$ cancels by subtraction. In fact, 


$$
\begin{aligned}
\left|C_{m, r, s}(x, i, k)\right| & <\sum_{s+2<j<r} \frac{s+1}{\left|x+i_{j}\right|} \\
& <(s+1)(r-s-1) \prod_{s+2<j<r} \frac{\left|i_{j}\right|+1}{\left|x+i_{j}\right|} .
\end{aligned}
$$

When $(i) \neq k$ as sets, we take advantage of whatever cancellation does occur to get

$$
\begin{aligned}
& \left|C_{m, r, s}(x, i, k)\right| \\
& <\frac{4 m r \Pi_{1<j<r}\left[\left(\left|i_{j}\right|+1\right)\left(\left|k_{j}\right|+1\right)\right]}{\Pi_{1<j<s+1}\left|x+i_{j}\right|^{(+1)} \Pi_{s+2<j<r}\left|x+i_{j}\right| \Pi_{s+2<j<r}\left|x+k_{j}\right|^{(+1)}}
\end{aligned}
$$

where $\left|x+i_{j}\right|^{(+1)}=1$ if $i_{j} \in k$ and $\left|x+i_{j}\right|^{(+1)}=\left|x+i_{j}\right|$ if $i_{j} \notin k$. The expression $\left|x+k_{j}\right|^{(+1)}$ is similarly interpreted. Then

$$
\begin{aligned}
& \left|H_{2 m, r, s}^{\prime}(u)\right| \\
& <\sum_{(i)} \sum_{k} \frac{P_{r, s}(i, k) \Pi_{1<j<r}\left(x+\eta_{j}\right)^{4 m} \varepsilon_{(i), k}(m)}{\Pi_{1<j<s+1}\left|x+i_{j}\right|^{2 m(+1)} \Pi_{s+2<j<r}\left|x+i_{j}\right|^{2 m-s} \Pi_{1<j<r}\left|x+k_{j}\right|^{2 m(+1)}}
\end{aligned}
$$

where $\varepsilon_{(i), k}(m)=1$ if $(i)=k$ as sets and $\varepsilon_{(i), k}(m)=m$, otherwise, and $P_{r, s}(i, k)$ is a polynomial of degree $<2 r-1$ in each $i_{j}$ and $k_{j}$ whose coefficients do not depend on $m$.

For $|u|>m$, the pairing argument which produced (1.11) can be applied to (1.16) to give

$$
\left|u^{\rho} H_{2 m, r, s}^{\prime}(u)\right|<\mathcal{O}(1) m(r \pi / u)^{2 m-\rho} \text { as } m \rightarrow \infty
$$

so that the integral for $|u|>r \pi$ is bounded for sufficiently large $m$.

For $|u|<m, u^{\rho}=\mathcal{O}(1)$. Now, we integrate over each of the $r$ intervals $[2 \pi l-\pi, 2 \pi l+\pi], l=(1-r) / 2,(3-r) / 2, \ldots,(r-1) / 2$. On the righthand side of (1.16), $m$ does not occur in the finite number of terms for which (i) $=k=\eta$ as sets so that the integral over these terms is $\theta(1)$ as $m \rightarrow \infty$. For each of the other terms, choose $\beta \in(i) \cup k$ so that $|\beta|>(r+1) / 2$. Now perform the pairing arguments except that the pairing for $\beta$ is now majorized by

$$
\left[a_{r}(\beta)\right]^{m}(r / 2|x+\beta|)^{m} \leqslant\left[a_{r}(\beta)\right]^{m}[r /(2 x+r+1)]^{m}
$$

(see (1.9)). As in (1.10), the sum is bounded by $\theta(1) \cdot m[r /(2 x+r+1)]^{m}$ as $m \rightarrow \infty$. The boundedness of the integral over $|x|<\frac{1}{2}$ follows. 
2. The limit of the $H_{2 m, r, s}(u)$. Next we consider the limit function for $H_{2 m, r, s}(u)$ and the nature of the convergence. After the arguments of the preceding lemmas, the limit is now transparent.

Definition 2.1. Let $\eta=\left\{\eta_{j}\right\}$ be as described in statement (1.4), and let (i) $=\eta$ mean that the indices agree as unordered sets. For $0<|x|<\frac{1}{2}$, $2 \pi x=u-2 \pi l$,

$$
Q_{r, s}(u)=(2 \pi)^{-s} \sum_{(i)=\eta} V(i) V_{1}(i) V_{2}(i) V^{-2}(\eta) \prod_{s+2<j<r}\left(x+i_{j}\right)^{s+1}
$$

and

$$
Q_{r, s}\left(2 \pi \eta_{j}+\pi\right)=\left[Q_{r, s}\left(2 \pi \eta_{j}+\pi+0\right)+Q_{r, s}\left(2 \pi \eta_{j}+\pi-0\right)\right] / 2,
$$

$j=0,1, \ldots, r\left(\eta_{0}=-(r+1) / 2\right)$.

Observe that the sum is vacuous if $l$ is not in the set $\eta$. Thus, $Q_{r, s}(u)=0$ for $|u|>r \pi$

Proposition 2.2. The relation

$$
\lim _{m \rightarrow \infty} H_{2 m, r, s}(u)=Q_{r, s}(u)
$$

holds for all $u$. Moreover, the convergence is uniform outside any neighbourhood of the points $2 \pi \eta_{j}+\pi, j=0,1, \ldots, r$.

Proof. By Lemma 1.4, the conclusion holds if $|u|>r \pi$. For $|u|<m$, $u \neq 2 \pi \eta_{j}+\pi(j=0,1, \ldots, r)$, a straightforward computation gives

$$
(2 \pi)^{s}\left(H_{2 m, r, s}(u)-Q_{r, s}(u)\right)=\left(N_{1}-N_{2}\right) / D
$$

where

$$
N_{1}=\sum_{(i) \neq \eta} V(i) V_{1}(i) V_{2}(i) V^{2}(\eta)
$$

$$
\begin{gathered}
\cdot \prod_{s+2<j<r}\left(x+i_{j}\right)^{s+1} \prod_{1<j<r}\left(\frac{x+S_{j}(\eta)}{x+i_{j}}\right)^{2 m}, \\
N_{2}=\sum_{\substack{(i)=\eta \\
k \neq \eta}} V(i) V_{1}(i) V_{2}(i) V^{2}(k) \\
\cdot \prod_{s+2<j<r}\left(x+i_{j}\right)^{s+1} \prod_{1<j<r}\left(\frac{x+S_{j}(\eta)}{x+k_{j}}\right)^{2 m}
\end{gathered}
$$

and

$$
D=V^{4}(\eta)+\sum_{k \neq \eta} V^{2}(k) V^{2}(\eta) \prod_{1<j<r}\left(\frac{x+S_{j}(\eta)}{x+k_{j}}\right)^{2 m}
$$


We employ the notation in the right-hand products to indicate that the pairing argument has taken place in each term.

Now, all $\left|\eta_{j}\right|<(r-1) / 2$ and, in each term of $N_{1}\left(N_{2}\right)$, there is a largest $\left|i_{j}\right|$ $\left(\left|k_{j}\right|\right)>(r+1) / 2$. Thus, if $0<|x|<\frac{1}{2}-\varepsilon$, then, as in the last step of the proof of Lemma 1.5,

$$
\left|\left(x+S_{j}(\eta)\right) /\left(x+i_{j}\right)\right|^{2 m}<\left[a_{r}\left(i_{j}\right)\right]^{m}[(r-2 \varepsilon) /(r+2 \varepsilon)]^{m}
$$

for that largest $\left|i_{j}\right|$ (similarly for the largest $\left.\left|k_{j}\right|\right)$. Then we easily obtain

$$
(2 \pi)^{s}\left(H_{2 m, r, s}(u)-Q_{r, s}(u)\right)=\mathcal{O}\left([(r-2 \varepsilon) /(r+2 \varepsilon)]^{m}\right)
$$

as $m \rightarrow+\infty$.

For a point of the form $u=2 \pi \eta_{j}+\pi(j=0,1, \ldots, r)$, observe that there are now two equal dominant terms corresponding to $\eta$ and $\eta-1 \equiv\left\{\eta_{j}-\right.$ $1: j=1, \ldots, r\}$. The restriction $(i) \neq \eta$ in $N_{1}$ becomes $(i) \neq \eta$ and $(i) \neq \eta$ -1 , which implies that in each term there is an $i_{j}$ such that $\left|x+i_{j}\right|=\left|\frac{1}{2}+i_{j}\right|$ $>(r+2) / 2$. In $N_{2}$, the restriction is $(i)=\eta$ or $(i)=\eta-1$ and $k \neq \eta$, $k \neq \eta-1$, insuring the existence of a $k_{j}$ such that $\left|x+k_{j}\right|>(r+2) / 2$. Therefore, the statement corresponding to $(2.5)$ includes $[r /(r+2)]^{m}$, so that (2.1) is $\theta\left([r /(r+2)]^{m}\right)$.

We exhibited (2.1) explicitly since we want to carry over the convergence to derivatives.

Proposition 2.3. For each integer $\rho>0$, the relation

$$
\lim _{m \rightarrow \infty} H_{2 m, r, s}^{(\rho)}(u)=Q_{r, s}^{(\rho)}(u)
$$

holds for all $u \neq 2 \pi \eta_{j}+\pi, j=0,1, \ldots, r$. The convergence is uniform outside any neighbourhood of the points $2 \pi \eta_{j}+\pi$. Moreover, for $|u|>m$,

$$
H_{2 m, r, s}^{(\rho)}(u)=\Theta\left(m^{\rho}(r \pi / u)^{m}\right) \text { as } m \rightarrow+\infty \text {. }
$$

Proof. The proof is necessarily technically involved. So, given that the pairing arguments and the arguments of the last proof are well established, we indicate how they can be used for this proof.

Consider a function of the form

$$
y(\alpha, \beta)=\prod_{1<j<r} \frac{\left(x+\eta_{j}\right)^{2 m-\alpha_{j}}}{\left(x+i_{j}\right)^{2 m-\beta_{j}}} .
$$

Clearly,

$$
\frac{d y(\alpha, \beta)}{d x}=\sum_{1<\gamma<r}\left[\left(2 m-\alpha_{\gamma}\right) y\left(\alpha+\delta_{j, \gamma}, \beta\right)-\left(2 m-\beta_{\gamma}\right) y\left(\alpha, \beta-\delta_{j, \gamma}\right)\right]
$$

where $\delta_{j, y}$ is the Kronecker $\delta$ function. Since this is a combination of 
functions of a similar type, an induction argument yields

$$
y^{(\rho)}(\alpha, \beta)=\sum_{\alpha^{\prime}, \beta^{\prime}} P\left(m, \alpha^{\prime}, \beta^{\prime}\right) y\left(\alpha^{\prime}, \beta^{\prime}\right)
$$

where the sum is finite $\left((2 r)^{\rho}\right.$ terms $)$ and $P\left(m, \alpha^{\prime}, \beta^{\prime}\right)$ is a polynomial of degree $\rho$ in $m$.

Applying this argument to the terms of $N_{1}$ in (1.18) and then applying our previous arguments, we obtain

$$
N_{1}^{(\rho)}=\theta\left(m^{\rho}[(r-2 \varepsilon) /(r+2 \varepsilon)]^{m}\right)
$$

as $m \rightarrow+\infty$ for $|u| \leqslant m$ and $0 \leqslant|x|<\frac{1}{2}-\varepsilon$, and

$$
N_{1}^{(\rho)}=\theta\left(m^{\rho}(m / u)^{m}\right)
$$

as $m \rightarrow \infty$ for $|u|>m$. Statements (2.7) and (2.8) are equally valid for $N_{2}$ in (2.3) and $D$ in (2.4).

Now, setting

$$
R=\left(N_{1}-N_{2}\right) / D, \quad N=R D,
$$

Leibniz's rule gives

$$
\begin{aligned}
R^{(\rho)} & =N^{(\rho)}-\sum_{0<\rho^{\prime}<\rho}\left(\begin{array}{c}
\rho \\
\rho^{\prime}
\end{array}\right) R^{\left(\rho-\rho^{\prime}\right)} D^{\left(\rho^{\prime}\right)}-D^{(\rho)} \\
& =\theta\left(m^{\rho}[(r-2 \varepsilon) /(r+2 \varepsilon)]^{m}\right)
\end{aligned}
$$

as $m \rightarrow \infty$ for $|u| \leqslant m, 0<|x|<\frac{1}{2}-\varepsilon$, and

$$
R^{(\rho)}=\theta\left(m^{\rho}(m / u)^{m}\right)
$$

as $m \rightarrow \infty$ for $|u|>r \pi$ by an induction argument.

We now give an alternate representation for $Q_{r, s}(u)$. This will make it possible to prove an identity useful in identifying the convergence class for cardinal Hermite spline interpolation.

LEMMA 2.4. If $(u-r \pi) / 2 \pi$ is not an integer,

$$
\begin{aligned}
& Q_{r, s}(u) \\
& =\frac{\sum_{n_{1}, \ldots, n_{r} ; n_{s+1}=0 ;\left|u+2 \pi n_{j}\right|<m} u^{-s} V\left(n_{1}, \ldots, n_{r}\right) \Pi_{1<j<r}\left(u+2 \pi n_{j}\right)^{j-1}}{\sum_{n_{1}, \ldots, n_{r}\left|u+2 \pi n_{j}\right|<m} V\left(n_{1}, \ldots, n_{r}\right) \Pi_{1<j<r}\left(u+2 \pi n_{j}\right)^{j-1}} .
\end{aligned}
$$

ProOF. For $|u|>r \pi$, the numerator in (2.10) is zero. For $|u|<r \pi$, we may follow the steps backward in the proofs of Lemmas 1.1 and 1.2 after noting that $\left|u+2 \pi n_{j}\right|<m$ implies that $n_{j}+l \in \eta$ where $l$ and $\eta$ are given by (1.1) and (1.4). 
LEMMA 2.5. The following relations hold:

$$
\sum_{s=0}^{r-1} u^{s} Q_{r, s}(u)= \begin{cases}1, & |u|<r \pi \\ \frac{1}{2}, & |u|=r \pi \\ 0, & |u|>r \pi\end{cases}
$$

and

$$
\sum_{s=0}^{r-1}(u+2 \pi k)^{s} Q_{r, s}(u)=\left\{\begin{array}{c}
0,-r \pi-2 \pi k<u \leqslant r \pi, k<0, \text { or } \\
-r \pi \leqslant u<r \pi-2 \pi k, k>0 \\
\frac{(-1)^{|k|-1} r !}{|k| !(r-|k|) ! 2}, \\
|u+2 \pi k|=r \pi, 0<|k| \leqslant r,|u| \leqslant r \pi .
\end{array}\right.
$$

Proof. The proof of (2.11) is evident from (2.10) and the fact that at the exceptional points $\left((u-r \pi) / 2\right.$ an integer) the function $Q_{r, s}$ is the average of the left- and right-hand limits.

For $k \neq 0$, looking at the numerator in equation (2.12) and arguing as in the proof of Lemma 1.1, we obtain

$$
\begin{aligned}
\sum_{s=0}^{r-1}(u & +2 \pi k)^{s} \sum u^{-s} V\left(n_{1}, \ldots, n_{r}\right) F\left(n_{1}, \ldots, n_{s}, 0, n_{s+2}, \ldots, n_{r}\right) \\
& =\sum_{s} \sum V\left(n_{1}, \ldots, n_{r}\right) F\left(n_{1}, \ldots, n_{s}, k, n_{s+2}, \ldots, n_{r}\right) \\
& =V\left(n_{1}^{\prime}, n_{2}^{\prime}, \ldots, n_{r}^{\prime}\right) V\left(u+2 \pi n_{1}^{\prime \prime}, \ldots, u+2 n_{r}^{\prime \prime}\right)
\end{aligned}
$$

where $\left\{n_{1}^{\prime}, \ldots, n_{r}^{\prime}\right\}$ is the set $n_{1}, \ldots, n_{r}$ in increasing order and $\left\{n_{1}^{\prime \prime}, \ldots, n_{r}^{\prime \prime}\right\}$ is the set $\left\{n_{1}^{\prime}, \ldots, n_{r}^{\prime}\right\}$ with 0 replaced by $k$.

If $-r \pi-2 \pi k<u \leqslant r \pi$ and $k<0$, or $-r \pi \leqslant u<r \pi-2 \pi k$ and $k>0$, then $k$ appears twice in the set $\left\{n_{1}^{\prime \prime}, \ldots, n_{r}^{\prime \prime}\right\}$ and the corresponding Vandermonde is zero.

If $u$ is such that $r \pi<u+2 \pi k<r \pi+2 \pi, k>0$, then the argument above yields the ratio

$$
\frac{(-1)^{k-1} V(0,1, \ldots, r-k-1, r-k+1, \ldots, r-1, r)}{V(0,1, \ldots, r-1)}=\frac{(-1)^{k-1} r !}{(r-k) ! k !} \text {. }
$$

Averaging as in the definition of $Q_{r, s}$, we obtain the result for $u=r \pi-2 \pi k$. Similarly, if $k<0$.

3. Uniform convergence. The convergence in Proposition 2.2 gives immediately the uniform limit of the fundamental splines for cardinal Hermite interpolation. Let 


$$
\mathscr{F}\left(Q_{r, s}\right)(x)=\frac{1}{2 \pi} \int_{-\infty}^{+\infty} Q_{r, s}(u) e^{i x u} d u
$$

be the Fourier transform of the function $Q_{r, s}$; then, by Proposition 2.2 and Lemma 1.4,

THEOREM 3.1. The relation

$$
\lim _{m \rightarrow \infty} L_{2 m, r, s}^{(\rho)}(x)=(-i)^{s} \mathscr{F}\left(Q_{r, s}\right)^{(\rho)}(x)
$$

holds uniformly in $x$ for each integer $\rho>0$.

In an effort to find the analog of Schoenberg's theorem for uniform convergence when interpolating the Fourier-Stieltjes transform of a measure, the formal process of replacing each $L_{2 m, r, s}$ by its Fourier transform, passing the sum through the integral, and then taking the limit suggests the following definition.

DEFINITION 3.2. Let $f(x)$ be defined by

$$
f(x)=\sum_{s=0}^{r-1} \frac{(-i)^{s}}{2 \pi} \int_{-r \pi}^{r \pi} Q_{r, s}(u) e^{i u x} d \beta_{s} \sim(u)
$$

where $\beta_{s}^{\sim}$ is the $2 \pi$-periodic extension of a bounded measure $\beta_{s}$ on $[-\pi, \pi)$.

Equation (3.1) is informative because of the formal limiting process outlined above, but it is also misleading until an application of Lemma 2.5 provides the "expected" result. Before we obtain the convergence result, we need two lemmas which will ensure that the proper kind of data is being interpolated.

LEMMA 3.3. The identity

$$
\sum_{l \in \mathbf{Z}}(u-2 \pi l)^{k} Q_{r, s}(u-2 \pi l)=\delta_{k, s}, \quad-\pi<u<\pi,
$$

holds for each $k, k=0,1, \ldots, r-1$.

Proof. Because of the behaviour of $H_{2 m, r, s}(u)$ for $|u|>m$, and the almost uniform convergence of $H_{2 m, r, s}(u)$ to $Q_{r, s}(u)$, it suffices to establish the identity for $H_{2 m, r, s}(u)$.

Since the denominator of $H_{2 m, r, s}(u)$ is independent of the translation involved in the identity, we only need to consider $H_{2 m, r, s}\left(\alpha_{2 m}(u)\right)$. Referring directly to the definition of $H_{r, s}\left(\alpha_{2 m}(u)\right)$ as a Hankel determinant and letting $l=-i_{s+1}$, the identity is obvious.

LEMMA 3.4. If $f(x)$ is defined by (3.1) and

$$
f_{s}(x)=\frac{1}{2 \pi} \int_{-\pi}^{\pi} e^{i u x} d \beta_{s}(u),
$$

then 


$$
f^{(j)}(\nu)=f_{j}(\nu), \quad \nu \in \mathbf{Z}, \quad j=0,1, \ldots, r-1 .
$$

Proof. Now

$$
f^{(j)}(x)=\sum_{s=0}^{r-1} \frac{(-i)^{s}}{2 \pi} \int_{-r \pi}^{r \pi} Q_{r, s}(u)(i u)^{j} e^{i u x} d \beta_{s}^{\sim}(u) .
$$

For $x=\nu$, we break the integration over integrals of the form $[2 \pi l-\pi, 2 \pi l$ $+\pi$ ), translate to $[-\pi, \pi)$ using periodicity, and use Lemma 3.3 to obtain

$$
\begin{aligned}
f^{(j)}(\nu) & =\sum_{s=0}^{r-1} \frac{i^{s+j}(-1)^{s}}{2 \pi} \int_{-\pi}^{\pi} \sum_{l}(u-2 \pi l)^{j} Q_{r, s}(u-2 \pi l) d \beta_{s} \\
& =f_{j}(\nu) .
\end{aligned}
$$

For $f \in C^{r-1}(\mathbf{R})$, the cardinal Hermite spline interpolating $f$ and its derivatives will be denoted by $f_{2 m, r}\left(f, f^{\prime}, \ldots, f^{(r-1)}\right)(x)$. The analogue of Schoenberg's theorem [16] follows.

THEOREM 3.5. If $f(x)$ is given by (3.1), then the relation

$$
\lim _{m \rightarrow \infty} \mathfrak{L}_{2 m, r}\left(f, f^{\prime}, \ldots, f^{(r-1)}\right)^{(\rho)}(x)=f^{(\rho)}(x)
$$

holds uniformly in $x$ on $\mathbf{R}$ for each integer $\rho \geqslant 0$.

Proof. Since the Cesaro means of the Fourier series of a bounded measure are weak* convergent to the periodic extension of the measure (see [1] and [13] where this was carried out for $r=1$ ), we have

$$
\begin{aligned}
\mathfrak{L}_{2 m, r}\left(f, f^{\prime}, \ldots, f^{(r-1)}\right)(x) & \\
& =\sum_{s=0}^{r-1} \frac{(-i)^{s}}{2 \pi} \int_{-\infty}^{+\infty} H_{2 m, r, s}(u) e^{i x u} d \beta_{s}^{\sim}(u)
\end{aligned}
$$

since $f^{(s)}(\nu)=f_{s}(\nu)$ by Lemma 3.4. Therefore,

$$
\begin{aligned}
\left|\mathscr{L}_{2 m, r}\left(f, f^{\prime}, \ldots, f^{(r-1)}\right)^{(\rho)}(x)-f^{(\rho)}(x)\right| \\
\quad<\sum_{s=0}^{r-1} \frac{1}{2 \pi} \int_{-\infty}^{+\infty}|u|^{\rho}\left|H_{2 m, r, s}(u)-Q_{r, s}(u)\right|\left|d \beta_{s}^{\sim}(u)\right| .
\end{aligned}
$$

Now, Lemma 1.4 and Proposition 2.2 show that the right-hand side tends to zero as $m \rightarrow+\infty$.

Observe that for $r=1$, Theorem 3.5 says that uniform convergence holds for $f(x)=(1 / 2 \pi) \int_{-\pi}^{\pi} Q_{1,0}(u) e^{i x u} d \beta \sim(u)$, where $Q_{1,0}(u)=1$ for $0<|u|<\pi$, $Q_{1,0}( \pm \pi)=\frac{1}{2}$, and $Q_{1,0}(u)=0$ otherwise. Thus, if $\beta$ has an atom at $-\pi$, then the formula automatically averages it at $\pi$ and $-\pi$ to compensate for the $\sin \pi x$ term of Schoenberg [16]. The next result shows that the situation is 
quite similar when $r>1$ and the measure has support in $[-r \pi, r \pi)$.

THEOREM 3.6. If

$$
f(x)=\frac{1}{2 \pi} \int_{-r \pi}^{r \pi} e^{i x u} d \beta(u)
$$

where $\beta$ is a bounded measure with support in $[-r \pi, r \pi)$, then for an appropriate constant $C$, the relation

$$
\lim _{m \rightarrow \infty} \varrho_{2 m, r}\left(f, f^{\prime}, \ldots, f^{(r-1)}\right)^{(\rho)}(x)=\left[f(x)+C(\sin \pi x)^{r}\right]^{(\rho)}
$$

holds uniformly for $x \in \mathbf{R}$ and any integer $\rho \geqslant 0$. Moreover, if $\beta$ does not have an atom at $-r \pi$, then $C \equiv 0$.

PRoOF. If $\beta=\beta_{1}+c \delta_{-m}$, then by (2.11), (2.12) and (3.3),

$$
\begin{aligned}
f(x)= & \sum_{s} \frac{(-i)^{s}}{2 \pi} \int_{-r \pi}^{r \pi} e^{i x u} Q_{r, s}(u)(i u)^{s} d \beta(u) \\
& +\frac{c}{4 \pi} \int_{-r \pi}^{r \pi} e^{i x u} d \delta_{-r \pi}(u) \\
= & \sum_{s} \frac{(-i)^{s}}{2 \pi} \int_{-r \pi}^{r \pi} e^{i x u} Q_{r, s}(u)\left(\sum_{k} i^{s}(u+2 \pi k)^{s} d \beta(u+2 \pi k)\right) \\
& +\frac{c}{4 \pi} \int_{-r \pi}^{r \pi} e^{i x u} \sum_{k=0}^{r}(-1)^{k}\left(\begin{array}{l}
r \\
k
\end{array}\right) d \delta_{(2 k-r) \pi}(u) .
\end{aligned}
$$

Hence, $f(x)$ differs from a function of the form (3.1) by an additive term of the form $C(\sin \pi x)^{r}$. The theorem follows from Theorem 3.5.

The remainder of this section is based on [13], and, for brevity, we will refer to the proofs therein whenever possible. We now consider a wider class of functions by permitting power growth on $\mathbf{R}$, but the convergence is weakened to uniform convergence on bounded sets.

For any sequence $y^{s}=\left\{y_{v}^{s}\right\}$ satisfying $\left|y_{\nu}^{s}\right|=\mathcal{O}\left(|\nu|^{\gamma}\right)$, there is a $2 \pi$-periodic distribution $T_{s}^{\sim}$ whose Fourier series is

$$
T_{s}^{\sim}=\sum_{\nu \in Z} y_{\nu}^{s} e^{i v t}
$$

where the series converges in the sense of tempered distributions. The Fourier transform of $T_{s}^{\sim}$ is given by

$$
\mathscr{F}\left(T_{s}^{\sim}\right)=\sum_{\nu \in Z} y_{\nu}^{s} \delta_{\nu}
$$

where $\delta_{\nu}$ is point evaluation at $\nu, \nu \in \mathbf{Z}$. The next theorem gives that the cardinal Hermite spline interpolating data of power growth is the Fourier transform of a tempered distribution (see equation (3.2) where the distribution is a measure). 
THEOREM 3.7. Let $\left(y^{0}, \ldots, y^{r-1}\right)$ be an r-tuple of sequences with power growth. Then

$$
\mathfrak{E}_{2 m, r}\left(y^{0}, \ldots, y^{r-1}\right)(x)=\sum_{s=0}^{r-1}(-i)^{s} \mathscr{F}\left(H_{2 m, r, s} T_{s}^{\sim}\right)(x)
$$

where $T_{s}^{\sim}$ is given by (3.4).

Proof. From equations (0.1), (0.2) and (3.5), the cardinal Hermite spline is visibly given as a sum of convolutions,

$$
\sum_{s=0}^{r-1} \mathscr{F}\left(T_{s}^{\sim}\right) * \mathscr{F}\left((-i)^{s} H_{2 m, r, s}\right) .
$$

Since all the derivatives of $H_{2 m, r, s}(u)$ exist and have at most power growth, a standard theorem from distribution theory provides the result (see [20, Chapter 30]).

When the data $y_{v}^{s}$ comes from the derivatives of certain functions of exponential type $r \pi$ with power growth on $\mathbf{R}$, the first part of this section and the results in [13] suggest some convergence results. However, there are pitfalls to avoid. First, the functions $Q_{r, s}(u)$ are not necessarily continuous at $2 \pi \eta_{j}+\pi, j=0,1, \ldots, r$, much less smooth. Secondly, the distributions can be thought of as (generalized) derivatives and the derivatives of $H_{2 m, r, s}(u)$ do not behave nicely at $2 \pi \eta_{j}+\pi, j=0,1, \ldots, r$. With this in mind, let $T_{0}, \ldots, T_{r-1}$ be distributions with support in $(-\pi, \pi)$ if $r$ is odd or support in $(0,2 \pi)$ if $r$ is even. Let $T_{0}^{\sim}, \ldots, T_{r-1}^{\sim}$ be their respective $2 \pi$-periodic extensions. Following (3.1), define

$$
f(x)=\sum_{s=0}^{r-1}(-i)^{s} \mathscr{F}\left(Q_{r, s} T_{s}^{\sim}\right)(x) .
$$

Because of Lemma 3.3 (see [23, Chapter 11]), we again obtain

$$
f^{(s)}(\nu)=\mathscr{F}\left(T_{s}\right)(\nu) .
$$

The method of proof for Theorem 2.2 [13] together with Proposition 2.2 and 2.3 and the fact that $Q_{r, s}(u)$ is smooth on the supports of $T_{s}^{\sim}$ yield the following result.

THEOREM 3.8. Let $f(x)$ be as defined in (3.7) with the supports of $T_{s}$ contained in $(-\pi, \pi)$ if $r$ is odd or in $(0,2 \pi)$ if $r$ is even. Then, for $\rho=0,1,2, \ldots$,

$$
\lim _{m \rightarrow \infty}\left|\rho_{2 m, r}\left(f, f^{\prime}, \ldots, f^{(r-1)}\right)^{(\rho)}(x)-f^{(\rho)}(x)\right|=0
$$

holds uniformly on bounded subsets of $\mathbf{R}$.

4. $l^{p} \oplus \cdots \oplus_{r} l^{p}$ data, $1<p<\infty$. This section contains results analogous to those produced in [6]. Since the methods of proof are similar, we 
shall usually only indicate how the results from $\$ 1$ can be employed.

The first lemma shows that the fundamental spline functions are uniformly dominated by an $L^{p}$-function, so that the convergence of Theorem 3.1 is also in $L^{p}(1<p<\infty)$.

LEMMA 4.1. The fundamental splines satisfy

$$
\left|L_{2 m, r, s}^{(\rho)}(x)\right|=\theta\left(\min \left(1,|x|^{-1}\right)\right), \quad m \rightarrow+\infty,
$$

for each $\rho, \rho=0,1, \ldots, r-1$.

Proof. The boundedness follows from Lemma 1.4 since

$$
\left|L_{2 m, r, s}^{(\rho)}(x)\right| \leqslant \frac{1}{2 \pi} \int_{-\infty}^{+\infty}|u|^{\rho}\left|H_{2 m, r, s}(u)\right| d u=\theta(1)
$$

as $m \rightarrow+\infty$. On the other hand, integration by parts and Lemma 1.5 combine to give

$$
\left|L_{2 m, r, s}^{(\rho)}(x)\right|=\left|\frac{1}{2 \pi x} \int_{-\infty}^{+\infty} e^{i x u} d\left(u^{\rho} H_{2 m, r, s}(u)\right)\right|=\mathcal{O}\left(|x|^{-1}\right),
$$

as $m \rightarrow+\infty$.

The above suggests that the cardinal Hermite spline interpolation operators, $l_{2 m, r}$, as mappings from $l^{p} \oplus \cdots \oplus_{r} l^{p}$ to $W^{p, r-1}(\mathbf{R})$ should converge. We first find a uniform bound for their norms.

THEOREM 4.2. The linear operator $\mathcal{E}_{2 m, r, s}$ defined on $y=\left\{y_{\nu}\right\} \in l^{p}(\mathrm{Z})$ by

$$
\mathcal{L}_{2 m, r, s} y(x)=\sum_{\nu \in \mathbf{Z}} y_{\nu} L_{2 m, r, s}(x-\nu)
$$

is a bounded linear operator from $l^{p}(\mathbf{Z})$ to $W^{p, r-1}(\mathbf{R}), 1<p<\infty$, with norm bounded independently of $\mathrm{m}$.

Proof. The proof is essentially the same as the proof of Theorem 3.1 in [6]. Indeed, for a finite sequence $y$ and for a function of compact support $g \in L^{p^{\prime}}(\mathbf{R}), 1 / p+1 / p^{\prime}=1$, the Fourier transform representation of $L_{2 m, r_{s},}$ Lemmas 1.4, 1.5 and 4.1, and the arguments cited above yield

$$
\begin{aligned}
& \left|\int_{-\infty}^{+\infty}\left(\varrho_{2 m, r, s} y(x)\right)^{(\rho)} g(x) d x\right| \\
& =\theta(1)\left(1+\|\mathcal{S C}\|_{p} \operatorname{Var}\left(u^{\rho} H_{2 m, r, s}(u)\right)\right)\|y\|_{l^{p}}\|g\|_{L^{p^{\prime}}} \\
& =\theta(1)\|y\|_{l l^{\prime}}\|g\|_{L^{p^{\prime}}} \text { as } m \rightarrow+\infty \text {, }
\end{aligned}
$$

where $\|\mathcal{H C}\|_{p}$ is the norm of the mixed Hilbert transform from $l^{p}(\mathbf{Z})$ to $L^{p}(\mathbf{R})$. Thus,

$$
\left\|\rho_{2 m, r, s} y(x)\right\|_{p, r-1} \leqslant \theta(1)\|y\|_{l p}, \quad m \rightarrow \infty .
$$

By the triangle inequality, we obtain immediately 
THEOREM 4.3. The operator $\mathfrak{l}_{2 m, r}$ is a bounded linear operator from $l^{p}$ $\oplus \cdots \oplus_{r} l^{p}$ to $W^{p, r-1}(\mathbf{R}), 1<p<+\infty$, with norm bounded independently of m.

The convergence Theorems 3.1 and 3.5 suggest the following operator which may be viewed as a generalization of the Whittaker cardinal series employed in [6].

Definition 4.4. Let $W_{r}$ be defined on $l^{p} \oplus \cdots \oplus_{r} l^{p}$ by

$$
W_{r}\left(y^{0}, \ldots, y^{r-1}\right)(x)=\sum_{s=0}^{r-1} \sum_{\nu \in \mathbf{Z}} y_{\nu}^{s}(-i)^{s} \mathscr{F}\left(Q_{r, s}\right)(x-v) \text {. }
$$

An argument similar to the proof of Theorem 4.2 shows that the series converges in $W^{p, r-1}(\mathbf{R})$ and that the operator $W_{r}$ is a bounded linear operator from $l^{p} \oplus \cdots \oplus_{r} l^{p}$ to $W^{p, r-1}(\mathrm{R}), 1<p<\infty$.

THEOREM 4.5. The relation

$$
\lim _{m \rightarrow \infty}\left\|\rho_{2 m, r}\left(y^{0}, \ldots, y^{r-1}\right)-W_{r}\left(y^{0}, \ldots, y^{r-1}\right)\right\|_{p, r-1}=0
$$

holds for each $\left(y^{0}, \ldots, y^{r-1}\right) \in l^{p} \oplus \cdots \oplus_{r} l^{p}, 1<p<\infty$.

Proof. This is similar to the proof of Theorem 3.3 in [6]. First, observe that by the triangle inequality we need only investigate each

$$
\left\|\int_{2 m, r, s} y^{s}(x)-\sum_{\nu \in Z} y_{v}^{s}(-i)^{s} \mathcal{F}\left(Q_{r, s}\right)(x-\nu)\right\|_{p, r-1},
$$

$s=0,1, \ldots, r-1$. The proof is completed by using Theorem 3.1, Lemma 4.1, Theorem 4.2, and the proof cited above.

A characterization of those functions in $W^{p, r-1}(\mathbf{R})$ which are the limits of cardinal Hermite splines would follow from a characterization of the functions $W_{r}\left(y^{0}, \ldots, y^{r-1}\right)$. In order to do this we must carry over to $W_{r}$ a result of Whittaker [21, p. 68] for the cardinal series.

Proposition 4.6. If $\Sigma_{v \neq 0}\left|y_{v}^{s} / \nu\right|<+\infty, s=0,1, \ldots, r-1$, then the series $W_{r}\left(y^{0}, \ldots, y^{r-1}\right)(x)$ is $(C, 1)$ summable to a function of the form

$$
f(x)=\sum_{s=0}^{r-1} \frac{(-i)^{s}}{2 \pi} \int_{-r \pi}^{r \pi} Q_{r, s}(u) e^{i u x} d f_{s}^{\sim}(u)
$$

where $f_{s} \in C\left(X_{\pi}\right), X_{\pi}=[-\pi, \pi)$ if $r$ is odd or $X_{\pi}=[0,2 \pi)$ if $r$ is even, and $f_{s}^{\sim}$ is its $2 \pi$-periodic extension .

Proor. Consult [21, pp. 65-68] for the basis of this proof. Since $\Sigma_{v \neq 0}\left|y_{v}^{s} / \nu\right|$ $<+\infty$, there are functions $f_{s} \in C\left(X_{\pi}\right)$ such that

$$
y_{r}^{s}=\frac{1}{2 \pi} \int_{X_{*}} e^{i m u} d f_{s}(u), \quad s=0,1, \ldots, r-1 .
$$


Now

$$
\begin{aligned}
\frac{(-i)^{s}}{2 \pi} \int_{-m \pi}^{m \pi} Q_{r, s}(u) e^{i x u} d f_{s}^{\sim}(u) & \\
& =\frac{(-i)^{s}}{2 \pi} \int_{X_{z}} \sum_{k \in \mathbf{Z}} Q_{r, s}(u+2 \pi k) e^{i x(u+2 \pi k)} d f_{s}(u) .
\end{aligned}
$$

By the choice of $X_{\pi}$, the function under the integral is continuous, $2 \pi$-periodic and of bounded variation in the variable $u$. Consider the Fourier coefficients of this function,

$$
\begin{aligned}
\frac{(-i)^{s}}{2 \pi} \int_{X_{\pi}} \sum_{k \in \mathbf{Z}} & Q_{r, s}(u+2 \pi k) e^{i x(u+2 \pi k)} e^{-i v u} d u \\
& =\frac{(-i)^{s}}{2 \pi} \int_{-m}^{r \pi} Q_{r, s}(u) e^{i(x-v) u} d u \\
& =(-i)^{s} \mathscr{F}\left(Q_{r, s}\right)(x-\nu) .
\end{aligned}
$$

Since the Cesaro means are of uniformly bounded variation [23, page 138], the arguments in Whittaker. [21, pages 67-68] give that the series $\sum_{\nu \in Z} y_{\nu}^{s}(-i)^{s} \mathcal{F}\left(Q_{r, s}\right)(x-\nu)$ is $(C, 1)$ summable to the function in (4.2). Combining these arguments for each $s, s=0,1, \ldots, r-1$, we obtain the result.

By Hölder's inequality for sequences, if $y^{s} \in l^{p}, 1<p<\infty$, then the condition in Proposition 4.6 holds. This leads to the following characterization theorem.

THEOREM 4.7. The following classes of functions are equivalent for $1<p<$ $+\infty$ :

(i) $W^{p, r-1}(\mathbf{R}) \cap\{f: f(x)$ of the form (4.1) $\}$,

(ii) $\left\{f: f(x)=W_{r}\left(y^{0}, \ldots, y^{r-1}\right)(x),\left(y^{0}, \ldots, y^{r-1}\right) \in l^{p} \oplus \cdots \oplus_{r} l^{p}\right\}$,

(iii) $\left\{f: f(x)=W^{p, r-1}-\lim \varrho_{2 m, r}\left(y^{0}, \ldots, y^{r-1}\right)(x),\left(y^{0}, \ldots, y^{r-1}\right) \in l^{p}\right.$ $\left.\oplus \cdots \oplus_{r} l^{p}\right\}$

Proof. The equivalence of (ii) and (iii) is Theorem 4.5. The containment of class (ii) in class (i) follows from Proposition 4.6. Finally, the containment of class (i) in class (ii) can be argued as in [7].

5. Examples. Special attention has previously been given to the cardinal spline interpolant of $\left(|\lambda| e^{i \tau}\right)^{x}$ (see [15, Lecture 3]). S. L. Lee [2] initiated a similar study for the cardinal Hermite spline interpolants, the so-called exponential Hermite Euler splines. For $|\lambda|=1$ and $-r \pi<\tau<r \pi$,

$$
e^{i \tau x}=\int_{-m \pi}^{r \pi} e^{i u x} d \delta_{\tau}(u)
$$

where $\delta_{\tau}(u)$ is point evaluation at $u=\tau$. This clearly falls under our 
discussion in §3. Thus, the cardinal Hermite splines interpolating $e^{i \tau x}$ and its derivatives to order $r-1$ converge uniformly on $\mathbf{R}$ to the function $e^{i \pi x}$.

Below we give the limit functions $Q_{r, s}(u)$ together with their Fourier transforms for $r=1,2,3$ and 4. It is interesting to note that discontinuities interior to the support can appear even when $s=0$.

Recall that $Q_{r, s}(u)=0$ if $|u|>r \pi$ and that we assign the average value at $2 \pi \eta_{j}+\pi, \eta_{j}=j-(r+1) / 2(j=0,1, \ldots, r)$.

$$
\begin{aligned}
& r=2, \eta=\left\{-\frac{1}{2}, \frac{1}{2}\right\} \text { : } \\
& Q_{2,0}(u)=(2 \pi-|u|) / 2 \pi, \quad Q_{2,1}(u)=(\operatorname{sgn} u) / 2 \pi, \\
& \mathscr{F}\left(Q_{2,0}\right)(x)=(\sin \pi x / \pi x)^{2}, \quad(-i) \mathscr{F}\left(Q_{2,1}(x)\right)=\sin ^{2} \pi x / \pi^{2} x ; \\
& r=3, \eta=\{-1,0,1\}: \\
& M_{3}(u)= \begin{cases}(u+3 \pi)^{2} / 2 & -3 \pi<u<\pi, \\
{\left[(u+3 \pi)^{2}-3(u+\pi)^{2}\right] / 2,} & -\pi<u<\pi, \\
(3 \pi-u)^{2} / 2, & \pi<u<3 \pi,\end{cases} \\
& Q_{3,0}(u)=(2 \pi)^{-2} M_{3}(u)-\frac{1}{8} M_{3}^{\prime \prime}(u), \\
& Q_{3,1}(u)=-(2 \pi)^{-2} M_{3}^{\prime}(u), \quad Q_{3,2}(u)=(2 \pi)^{-2} M_{3}^{\prime \prime}(u) / 2, \\
& \mathscr{F}\left(Q_{3,0}\right)(x)=(\sin \pi x / \pi x)^{3}\left[1+\pi^{2} x^{2} / 2\right] \text {, } \\
& (-i) \mathscr{F}\left(Q_{3,1}\right)(x)=(\sin \pi x / \pi x)^{3} x \text {, } \\
& (-i)^{2} \mathscr{F}\left(Q_{3,2}\right)(x)=(\sin \pi x / \pi x)^{3}\left(x^{2} / 2\right) ; \\
& r=4, \eta=\left\{-\frac{3}{2}, \frac{1}{2}, \frac{1}{2}, \frac{3}{2}\right\} \text { : }
\end{aligned}
$$

$$
\begin{aligned}
& M_{4}(u)= \begin{cases}(u+4 \pi)^{3} / 6, & -4 \pi<u<-2 \pi, \\
{\left[(u+4 \pi)^{3}-4(u+2 \pi)^{3}\right] / 6,} & -2 \pi<u<0, \\
{\left[(4 \pi-u)^{3}-4(2 \pi-u)^{3}\right] / 6,} & 0<u<2 \pi, \\
(4 \pi-u)^{3} / 6, & 2 \pi<u<4 \pi,\end{cases} \\
& Q_{4,0}(u)=(2 \pi)^{-3} M_{4}(u)-\frac{1}{6} M_{4}^{\prime \prime}(u)(2 \pi)^{-1}, \\
& Q_{4,1}(u)=-(2 \pi)^{-3} M_{4}^{\prime}(u)+(2 \pi)^{-1} \frac{1}{6} M_{4}^{\prime \prime \prime}(u), \\
& Q_{4,2}(u)=(2 \pi)^{-3} M_{4}^{\prime \prime}(u) / 2, \quad Q_{4,3}(u)=-(2 \pi)^{-3} M_{4}^{\prime \prime \prime}(u) / 6, \\
& \mathscr{F}\left(Q_{4,0}\right)(x)=(\sin \pi x / \pi x)^{4}\left[1+2 \pi^{2} x^{2} / 3\right], \\
& (-i) \mathscr{F}\left(Q_{4,1}\right)(x)=(\sin \pi x / \pi x)^{4}\left[x+2 \pi^{2} x^{3} / 3\right],
\end{aligned}
$$




$$
\begin{aligned}
& (-i)^{2} \mathscr{F}\left(Q_{4,2}\right)(x)=(\sin \pi x / \pi x)^{4}\left(x^{2} / 2\right), \\
& (-i)^{3} \mathscr{F}\left(Q_{4,3}\right)(x)=(\sin \pi x / \pi x)^{4}\left(x^{3} / 6\right) .
\end{aligned}
$$

Notice that the functions $M_{r}(u)$ are $B$-splines on intervals of length $2 \pi$.
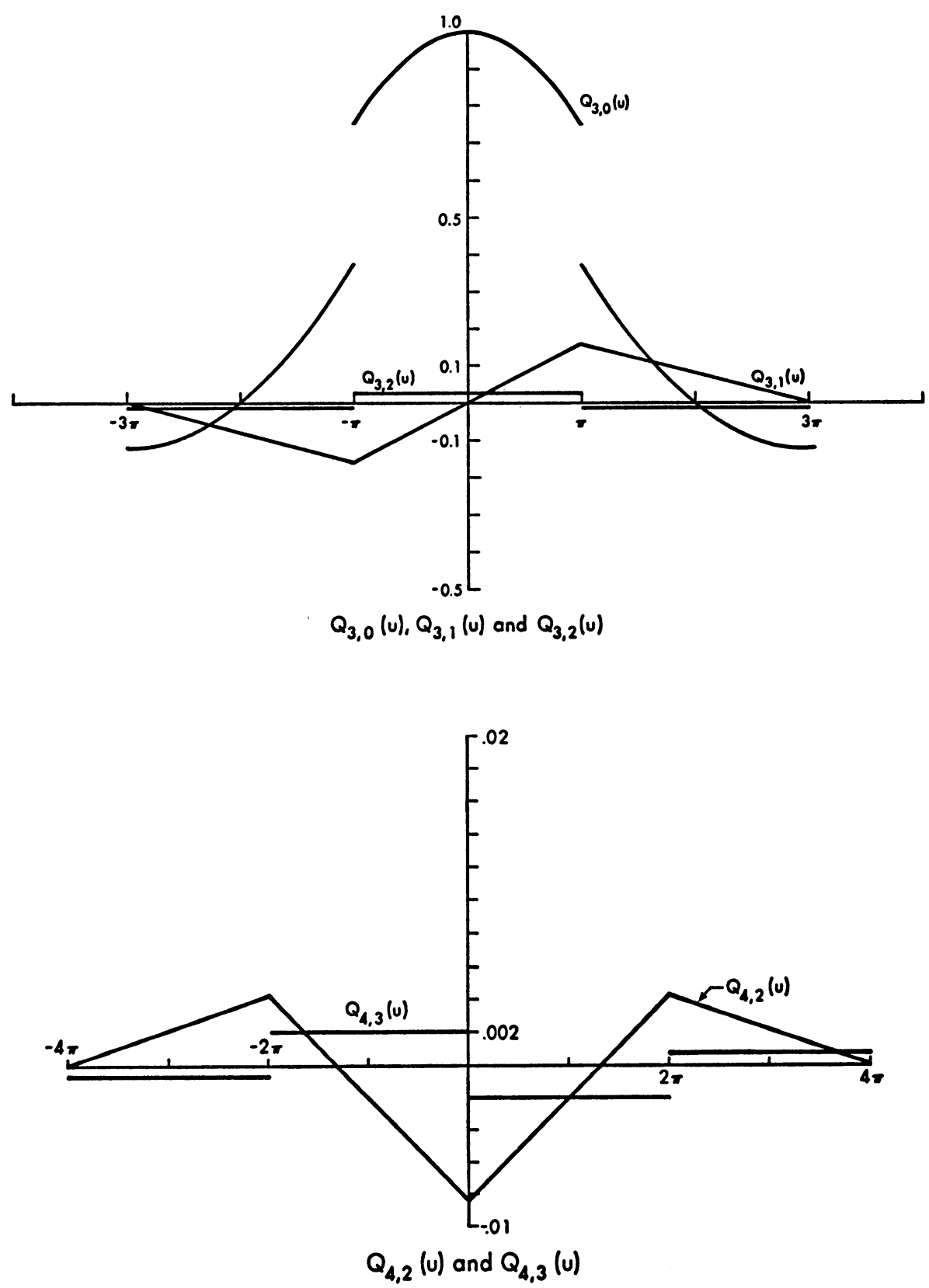


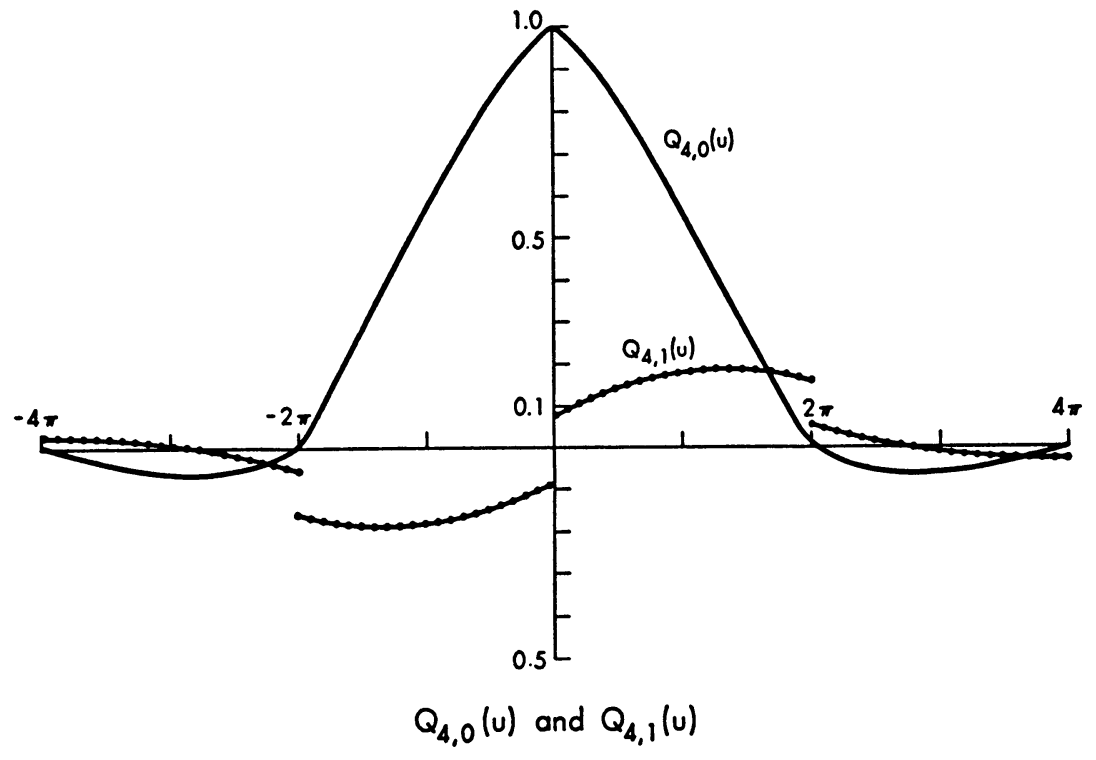

\section{REFERENCES}

1. Richard R. Goldberg, Restrictions of Fourier transforms and extension of Fourier sequences, J. Approximation Theory 3 (1970), 149-155. MR 41 \# 5885.

2. S. L. Lee, Exponential Hermite Euler splines, J. Approximation Theory 18 (1970), 205-212.

3. _. Fourier transforms of B-splines and fundamental splines for cardinal Hermite interpolations, Proc. Amer. Math. Soc. 57 (1976), 291-296.

4. Peter R. Lipow and I. J. Schoenberg, Cardinal interpolation and spline functions. III. Cardinal Hermite interpolation, J. Linear Algebra Appl. 6 (1973), 273-304.

5. M. Marsden and R. Mureika, Cardinal spline interpolation in $L_{2}$, Illinois J. Math. 19 (1975), 145-147. M:R 51 \# 13529.

6. M. J. Marsden, F. B. Richards and S. D. Riemenschneider, Cardinal spline interpolation operators on $l^{p}$ data, Indiana Univ. Math. J. 24 (1975), 677-689. MR 52 \#3807.

7. __ Erratum to "Cardinal spline interpolation operators on $l^{p}$ data", Indiana Univ. Math. J. 25 (1976), 919. MR 53 \# 13926.

8. M. J. Marsden and S. D. Riemenschneider, Convergence results for cardinal Hermite splines, Approximation Theory II (G. G. Lorentz, C. K. Chui, and L. L. Schumaker, Editors), Academic Press, New York, 1976, pp. 457-462.

9. D. J. Newman, Some remarks on the convergence of cardinal splines, MRC Report \#1474, Math. Research Center, Univ. of Wisconsin, Madison, 1974.

10. Franklin Richards, Uniform spline interpolation operators in $L_{2}$, Hlinois J. Math. 18 (1974), 516-521. MR 50 \# 10620.

11. _ The Lebesgue constants for cardinal spline interpolation, J. Approximation Theory 14 (1975), 83-92. MR 52 \#6254.

12. Franklin B. Richards and I. J. Schoenberg, Notes on spline functions. IV. A cardinal spline analogue of the theorem of the brothers Markov, Israel J. Math. 16 (1973), 94-102.

13. S. D. Riemenschneider, Convergence of interpolating cardinal splines: Power growth, Israel J. Math. 23 (1976), 339-346.

14. I. J. Schoenberg, Notes on spline functions. I. The limits of the interpolating periodic spline functions as their degree tends to infinity, Nederl. Akad. Wetensch. Proc. Ser. A $75=$ Indag. Math. 34 (1972), 412-422. MR 47 \#5492. 
15. Cardinal spline interpolation, Regional Conf. Series in Appl. Math., no. 12, S.I.A.M., Philadelphia, Pa., 1973.

16. Notes on spline functions. III. On the convergence of the interpolating cardinal splines as their degree tends to infinity, Israel J. Math. 16 (1973), 87-93.

17. _ Cardinal interpolation and spline functions. VII. The behavior of cardinal spline interpolants as their degree tends to infinity, J. Analyse Math. 27 (1974), 205-229.

18. - On remainders and the convergence of cardinal spline interpolation for almost periodic functions, Studies in Spline Functions and Approximation Theory (S. Karlin, C. A. Micchelli, A. Pinkus, and I. J. Schoenberg, Editors), Academic Press, New York, 1976, pp. 277-303.

19. On spline interpolation at all integer points of the real axis, Mathematica (Cluj) 10 (33) (1968), 151-170. MR 38 \#6274.

20. F. Trèves, Topological vector spaces, distributions and kernels, Academic Press, New York, 1967. MR 37 \# 726.

21. J. M. Whittaker, Interpolatory function theory, Cambridge Univ. Press, London, 1935.

22. A. H. Zemanian, Distribution theory and transform analysis, McGraw-Hill, New York, 1965. MR 31 \# 1556.

23. A. Zygmund, Trigonometric series. Vol. I, 2nd ed., reprinted with corrections, Cambridge Univ. Press, London and New York, 1968. MR 38 \#4882.

Department of Mathematics and Statistics, University of Pittsburgh, Pittsburgh, Pennsylvania 15260

Department of Mathematics, University of Alberta, Edmonton, Alberta, Canada T6G 2GI 